\title{
Concepciones sobre ser músicx y ser músicx profesional de Ixs aspirantes a las carreras de Música de la FDA-UNLP
}

Conceptions about being a musician and being a professional musician from enrolled students of Music majors of FDA-UNLP

Juan Félix Pissinis

https://orcid.org/0000-0001-8906-9263

juanfelixpissinis@gmail.com

Laboratorio para el Estudio de la Experiencia

Musical | Facultad de Artes | Universidad

Nacional de La Plata

\author{
Santiago Peluffo \\ https://orcid.org/0000-0003-4890-6840 \\ santiagoandrespeluffo@gmail.com \\ Laboratorio para el Estudio de la Experiencia \\ Musical | Facultad de Artes | Universidad \\ Nacional de La Plata
}

\author{
Isabel Cecilia Martínez \\ https://orcid.org/0000-0002-1837-5957 \\ isabelmartinez@fba.unlp.edu.ar \\ Laboratorio para el Estudio de la Experiencia \\ Musical | Facultad de Artes | Universidad \\ Nacional de La Plata
}

\section{RESUMEN}

Las condiciones de ingreso irrestricto que presentan las carreras de Música de la Facultad de Artes de la Universidad Nacional de La Plata (FDA-UNLP), han propiciado un aumento progresivo del número de estudiantes ingresantes en los últimos años, con las complejidades que conlleva la realización de prácticas de enseñanza-aprendizaje en condiciones de masividad. Es por ello que resulta de interés indagar en el estado actual de lxs aspirantes a ingresantes, con el fin de ampliar la base de información sobre la que se sustentan dichas prácticas.

En esta dirección, realizamos un estudio de carácter exploratorio que consistió en una serie de entrevistas semi-estructuradas a aspirantes a las carreras al momento de inscripción a las mismas, cuyo guión temático abordó diversos aspectos que tienen que ver con la experiencia musical previa de lxs ingresantes, sus concepciones en torno al ser músicx y sus expectativas en vinculación con la carrera en que se inscribieron.

En este trabajo presentamos resultados acerca de las dimensiones sobre las cuales Ixs aspirantes a las carreras de Música modelan sus concepciones acerca de ser músicx y ser músicx profesional, dimensiones que abarcan aspectos diversos y a veces son valoradas de maneras contrastantes por Ixs participantes.

\section{PALABRAS CLAVE}

Ingreso Universitario, Estudiantes ingresantes, Concepciones sobre ser músicx. 


\section{KEY WORDS}

University Entrance, Incoming Students, Conceptions about being a musician

\section{ABSTRACT}

The unrestricted admission conditions presented by the Music majors of the Faculty of Arts of the National University of La Plata (FDA-UNLP), have led to a progressive increase in the number of incoming students in recent years, with the complexities involved in performing teaching-learning practices in massive conditions. That is why it is of interest to investigate the current status of enrolled students, in order to expand the knowledge on which these practices are based.

In this direction, we carried out an exploratory study that consisted of a series of semi-structured interviews with students at the time of enrollment, whose thematic script addressed various aspects regarding the prior musical experience of the students, their conceptions about being a musician and their expectations in relation to the degree in which they enrolled.

In this paper we present results about the dimensions on which enrolled students of Music majors model their conceptions about being a musician and being a professional musician, dimensions that encompass diverse aspects and are sometimes valued in contrasting ways by the participants. 


\section{INTRODUCCIÓN}

El aumento de la cantidad de estudiantes dentro de las aulas universitarias a costa de una peor calidad educativa, no equivale a un sistema educativo inclusivo. Además de propiciar condiciones que permitan acceder a la Universidad a poblaciones que, por diversos motivos, no contaban con esa posibilidad, una lógica inclusiva implica que la Universidad se transforme para poder atender a la pluralidad en los procesos de enseñanza-aprendizaje, alentando la participación y promoviendo los logros de todas las personas que ingresan a ella (Blanco Guijarro, 2008). Un sistema educativo inclusivo podrá trabajar en incrementar su espectro de posibilidades una vez que haya reconocido a Ixs destinatarixs de esa educación, con sus características colectivas e individuales, como verdaderxs protagonistas del proceso educativo, trascendiendo así la invisibilización epistémica del lxs mismxs (Pissinis y Martínez, 2019b).

En la Facultad de Artes de la Universidad Nacional de La Plata (FDAUNLP), el reemplazo en 2006 de una prueba de desempeño específica para cada una de las orientaciones de las carreras de Música que restringía el ingreso a las mismas, por una asignatura anual de carácter propedéutico para cada orientación, y la apertura de una séptima orientación en 2008, han promovido un gradual aumento del número de ingresantes a dichas carreras, potenciando las complejidades que acarrean las condiciones de masividad para las prácticas de enseñanza-aprendizaje.

Por ello, en un trabajo previo (Pissinis y Martínez, 2019b) resaltamos el valor de la indagación sobre los antecedentes musicales de Ixs estudiantes ingresantes a las carreras de Música de dicha Facultad: sus 
concepciones, saberes, expectativas, habilidades. Planteamos, a partir de los resultados de estudios anteriores (Pissinis y Martínez, 2018; 2019a; Martínez, 2018; Valles et al, 2018), que dicha información organizada puede servir de punto de partida para revisar las situaciones de enseñanza-aprendizaje en vistas a mejorar las conexiones entre los contenidos de la formación musical y el estado actual de Ixs estudiantes (Vigotsky, 1978), y fomentar la adjudicación de un mayor grado de significatividad en los aprendizajes (Ausubel, Novak, y Hanesian, 1978) por parte de éstxs.

Presentamos aquí resultados de un primer trabajo de indagación en las concepciones de Ixs aspirantes a las carreras de Música de la FDA-UNLP, realizado a través de entrevistas exploratorias, donde se abordan, entre otros aspectos, sus concepciones acerca de ser músicx y ser músicx profesional. Estos resultados complementan otros ya presentados respecto de las expectativas de aprendizaje y desarrollo de proyectos futuros que manifestaron Ixs participantes en vinculación a la carrera en que se inscribieron (Pissinis, Peluffo y Martinez, 2020).

\section{Presentamos aquí resultados de un primer trabajo de inda- gación en las concepciones de Ixs aspirantes a las carreras de Música de la FDA-UNLP, realizado a través de entrevistas ex- ploratorias, donde se abordan, entre otros aspectos, sus con- cepciones acerca de ser músicx y ser músicx profesional.}

\section{OBJETIVO}

Realizar una indagación exploratoria acerca de cómo son las concepciones con respecto a ser músicx y ser músicx profesional que poseen Ixs aspirantes a las carreras de Música de la FDA-UNLP.

\section{METODOLOGÍA}

Dado que el auto-reporte ha demostrado ser uno de los medios más fiables para indagar la experiencia humana y que el interés de este trabajo está centrado en la dimensión subjetiva de Ixs participantes, una metodología cualitativa se presenta como la más adecuada, ya que pone el foco allí.

\section{PARTICIPANTES}

Participaron del estudio 39 personas, quienes se inscribieron a las carreras de Música de la FDA-UNLP entre noviembre y diciembre de 2019 para el Ingreso 2020. 


\section{Participaron del estudio 39 personas, quienes se inscribieron a las carreras de Música de la FDA-UNLP entre noviembre y diciembre de 2019 para el Ingreso 2020.}

\section{TÉCNICAS DE PRODUCCIÓN DE LA INFORMACIÓN}

Se realizaron 39 entrevistas individuales semi-estructuradas El guión temático estuvo orientado a indagar en diferentes aspectos que tienen que ver con la experiencia previa, concepciones y expectativas de Ixs aspirantes en relación a la música y las carreras en las que se inscribieron.

\section{PROCEDIMIENTO}

Lxs participantes fueron contactadxs personalmente, inmediatamente después de realizar el trámite de inscripción. Allí fueron invitadxs a participar, previa explicación y contextualización de los objetivos y condiciones del estudio. Las entrevistas fueron realizadas en ese mismo momento, en un lugar cómodo en los pasillos o el patio de la Facultad.

\section{ANÁLISIS DE DATOS}

La técnica de análisis elegida es la teoría fundamentada.

Se realizó un análisis individual de las transcripciones de las entrevistas buscando unidades de significado relevantes para la investigación y realizando una codificación abierta, aplicando el procedimiento de comparación constante.

Luego se realizó una revisión grupal de las codificaciones propuestas de manera individual, que dio lugar a una codificación en común y una organización jerárquica de las categorías.

\section{RESULTADOS}

Mediante el análisis de las entrevistas hemos identificado diferentes dimensiones a partir de las cuales Ixs participantes articulan sus concepciones sobre ser músicx y ser músicx profesional.

Estas dimensiones no son excluyentes, sino que muchas veces surgieron conjuntamente en una misma respuesta. A su vez, si bien en la mayoría de los casos fueron mencionadas como un aspecto importante del ser músicx o músicx profesional, algunas de estas dimensiones fueron consideradas irrelevantes o poco importantes por Ixs participantes.

Por otro lado, hemos agrupado estas dimensiones en función de si 
remiten a una instancia de carácter más personal o individual, o si son aspectos más vinculados a la relación del individuo con Ixs otrxs o con el contexto.

\section{CONCEPCIONES SOBRE SER MÚSICX}

Las dimensiones de carácter personal que entraron en juego a la hora de considerar a alguien como músicx son: Saberes Prácticos, Saberes Teóricos, Compromiso Afectivo, Actitud, Creatividad y Autoconsideración.

\section{Las dimensiones de carácter personal que entraron en juego a la hora de considerar a alguien como músicx son: Saberes Prácticos, Saberes Teóricos, Compromiso Afectivo, Actitud, Creatividad y Autoconsideración.}

Por Saberes Prácticos entendemos la posesión de saberes performativos relativos a la práctica musical, como saber tocar instrumentos, cantar, componer. Si bien la gran mayoría de las veces Ixs participantes remiten a esta dimensión destacando su importancia, con expresiones como: «soy músico porque toco y compongo», hubo algunos casos en que fue presentada como una dimensión poco relevante: «no necesitás ni cantar, ni saber componer una letra, nada. Necesitás alguien que te ayude y te hace músico». Los Saberes Teóricos, a su vez, implican la posesión conocimientos derivados de la teoría musical, como conocimientos sobre escalas, acordes, tonalidad: «obviamente, tenés que tener una teoría, lo básico».

Por otro lado, el Compromiso Afectivo tiene que ver con la disposición afectiva hacia la música, sentir amor, pasión por ella, disfrutarla: «tenés que sentir la música en el alma», «la pasión es la esencia». La Actitud refiere a la adopción de una actitud dedicada y perseverante ante la práctica musical, ser constante: hay que «ponerle ganas, dedicarle tiempo», «tenés que tener constancia, sentarte a tocar seguido». La dimensión Creatividad se vincula con ser una persona imaginativa, creativa, original: unx músicx «debería ser bastante creativo, bastante original», «tiene que tener imaginación». Finalmente, la Autoconsideración implica considerarse unx mismx como músicx, sentirse músicx, considerar que lo que unx hace es música: «A mí me dicen "vos sos músico". Yo no estudié, voy a estudiar ahora, entonces no sé, yo sé tocar, no sé si soy músico. Y creo que eso lo tiene que decidir la persona... si se considera música, músico».

Entre las dimensiones vinculadas a la relación del individuo con Ixs otrxs o con el contexto encontramos: Expresividad, Consciencia Social, 
Fama y Contexto. La Expresividad requiere saber expresarse a través de la música, poder transmitir sentimientos, llegarle a otras personas, conectarse con ellas: para ser músicx es necesario «saber expresarse, hacerle llegar algo a alguien», "pasa por una interpretación o por una creación que llegue a alguien más, apenas exista esa conexión ya es un músico». La Consciencia Social implica tener consciencia sobre el rol social que unx cumple como músicx y la responsabilidad que desempeñar ese rol conlleva: «si un músico se sube a un escenario, saber que no es que estás tocando y nada más, sino que estás transmitiendo un mensaje hacia un público, ser consciente. El músico tiene muchos factores, más allá de hacer música. Tiene una responsabilidad civil, moral». La Fama, es decir, tener cierto reconocimiento popular, fue referenciada como una dimensión irrelevante para la condición de ser músicx: «no es necesario ser reconocido. Con tal de hacer música ya sos músico, de ahí a ser reconocido, o famoso, es otra cosa». Finalmente, la dimensión del Contexto, entendida en diferentes niveles, como lo socio-histórico-cultural en sentido amplio, las prácticas estilísticas específicas, el nivel situacional: «hará 50 años atrás, la persona que no sabía cantar ni tocar una guitarra no era músico. Hoy no es necesariamente saber cantar o tocar un instrumento, realmente nada, hoy en día la música varía muchísimo y cambió un montón», hay «géneros en los que no necesitás saber nada para ser músico».

Parece ser que el grupo que contiene las dimensiones relativas a una instancia de carácter personal o individual es el que tiene más peso en la configuración de la idea de ser músicx que poseen Ixs participantes, al menos considerando la frecuencia con la que se hacen presentes en su discurso. A su vez, dentro de este grupo, las dos dimensiones que se destacan en cantidad de apariciones por sobre el resto son las de Saberes Prácticos y Compromiso Afectivo, en ese orden. Vale recordar que algunas dimensiones, como es el caso de los Saberes Prácticos, fueron mencionadas tanto para destacar su importancia, como para desdeñarlas.

Por otro lado, la dimensión correspondiente a la Expresividad, incluida en el grupo de dimensiones que atañen al vínculo del individuo con Ixs otrxs y el contexto, aparece tan frecuentemente como la dimensión de Compromiso Afectivo, e incluso varias veces interrelacionada con ésta en una misma respuesta. Si bien aquí la categoría Expresividad involucra un nivel interpersonal, se refiere a una característica del individuo.

\section{CONCEPCIONES SOBRE SER MÚSICX PROFESIONAL}

En lo que respecta a las dimensiones que surgieron alrededor de las concepciones de ser músicx profesional que poseen Ixs participantes, entre las de carácter personal volvieron a aparecer cuatro que 
ya habían surgido en relación al ser músicx: Saberes Prácticos, Saberes Teóricos, Compromiso Afectivo y Actitud. Sin embargo, en este caso se invierten las frecuencias de aparición, siendo la dimensión Saberes Teóricos la más frecuente de ellas, seguida por la Actitud. El Compromiso Afectivo, que tanta preponderancia tuvo en la idea de ser músicx, apareció en una sola respuesta en relación al ser múscix profesional. En esta instancia, cada dimensión fue mencionada como un aspecto relevante.

Entre las dimensiones vinculadas a la relación del individuo con Ixs otrxs o con el contexto sobre las que se articula la idea de ser músicx profesional encontramos dos que se repiten respecto al ser músicx, aunque nuevamente, con su frecuencia totalmente invertida respecto de aquel: la Expresividad, en este caso, es la menos aludida del grupo, en tanto que la Fama es la dimensión más aludida: «"profesionales" es como igual a "famosos"» (en algunos pocos casos, ésta fue considerada como una variable no importante).

\section{""profesionales" es como igual a"famosos"» (en algunos pocos casos, ésta fue considerada como una variable no importante).}

Además, surgieron otras tres dimensiones: Estudios Formales, Medio de Vida y Docencia. La posesión de Estudios Formales, el tener un título de grado, o al menos haber transitado por instituciones de formación musical, fue la segunda dimensión más frecuente de este grupo, mencionada tanto como un aspecto determinante de la profesionalidad: "profesional es el que estudia la licenciatura en música», como desestimando su importancia: «hay muchos profesionales que no han estudiado y son músicos profesionales». Si bien hubo 2 personas que plantearon ambas consideraciones respecto de esta dimensión, la mayoría se inclinó hacia la segunda.

La profesionalidad como Medio de Vida, que tiene que ver con desarrollar la actividad como principal fuente de ingreso económico, también fue mencionada tanto como una dimensión importante: "cuando se habla de profesional se habla de una persona que cobra y vive de eso», como para desestimarla: «uno dice "es profesional" cuando uno hace las cosas bien, no cuando a uno se le paga», aunque en este caso en proporciones bastante equilibradas.

La otra dimensión mencionada en relación a la profesionalidad del músicx, con menos frecuencia que las dos anteriores, pero siempre destacando su importancia, es la Docencia, saber enseñar, transmitir conocimientos: para ser músicx profesional hay que «ser docente, profesor, poder llegar a tus alumnos, generar vínculos».

En el caso de la concepción sobre ser músicx profesional que poseen Ixs participantes, pareciera haber mucho mayor equilibrio en la preponderancia que tienen las dimensiones de uno y otro grupo. 


\section{AUTOPERCEPCIÓN COMO MÚSICXS}

Por otro lado, aproximadamente 2 de cada 5 participantes dijeron no considerarse a sí mismxs músicxs. De esos casos, solo en un tercio de ellos parece ser consistente la autopercepción de cada participante en cuanto a ser músicx o no en relación con los criterios descriptos para considerar a una persona música y a las propias experiencias musicales previas, narradas al comienzo de la entrevista.

Entre los restantes casos, se dan diversas situaciones. Algunas personas no argumentan por qué no se consideran músicas, aunque, aparentemente, no estarían aplicando a su propia experiencia previa el mismo criterio que aplicarían en otras personas, al menos según sus respuestas anteriores. Otras arguyen no considerarse músicas esgrimiendo un criterio que no habían mencionado anteriormente, sin percatarse de este hecho (al menos explícitamente). Algunos casos reconocen que aunque hacen música, e incluso se han presentado con público en vivo, no se consideran a sí mismxs músicxs. En otro caso, alguien manifestó no considerarse a sí mismx músicx, por «temas más personales y emocionales», «de confianza».

\section{DISCUSIÓN}

Es de notar lo contrastante de ciertas tendencias que se presentan en torno a cuáles son las dimensiones que más pesan en sus concepciones sobre ser músicx en función de si lxs participantes las proyectan hacia otra persona o hacia sí mismxs.

\section{Es de notar lo contrastante de ciertas tendencias que se pre- sentan en torno a cuáles son las dimensiones que más pesan en sus concepciones sobre ser músicx en función de si lxs par- ticipantes las proyectan hacia otra persona o hacia sí mismxs.}

Lamont (2002) afirma que la identidad musical no se construye a partir de la adquisición de un conjunto de habilidades técnicas preestablecidas, sino más bien a partir de una autoevaluación con respecto a otras personas significativas. Para Salvador (2014), estas identidades son fluidas y evolucionan, de modo que cualquier persona puede tener en un mismo momento distintas identidades de músicx en diferentes contextos, las cuales pueden cambiar con el tiempo.

Los hallazgos surgidos a partir del análisis de estas entrevistas parecieran ir en esta dirección. Por un lado, hubo varios casos que, además de responder que no se consideraban a sí mismxs músicxs y argumentar o no el porqué de esa consideración, explicitaron entender que «todavía» no lo son pero están «en proceso» de serlo, mostrando una cierta consciencia sobre la mutabilidad de esa condición. 
hubo varios casos que, además de responder que no se consideraban a sí mismxs músicxs y argumentar o no el porqué de esa consideración, explicitaron entender que «todavía» no lo son pero están «en proceso» de serlo, mostrando una cierta consciencia sobre la mutabilidad de esa condición.

Por el otro, parecen convivir en varias de las personas participantes dos concepciones diferentes del ser músicx, una más amplia y otra más estricta. Cuando hablan de un otrx como músicx, el considerable hincapié puesto sobre dimensiones como el Compromiso Afectivo o la Expresividad podría vincularse con la idea de musicar (Small, 1999), e incluso con la de musicalidad comunicativa (Malloch y Trevarthen, 2009). En cambio, cuando el foco está puesto en unx mismx, no en todos los casos, pero sí en una considerable proporción, pareciera estar operando una lógica más vinculada a las ontologías de música y ser músicx privilegiadas por el modelo conservatorio (Holguín y Shifres, 2015).

Con respecto a la Fama, la dimensión más veces mencionada en relación a ser músicx profesional, es de notar que los ejemplos nombrados, en los casos en que los hubo, son todos intérpretes varones de dos de los géneros que más peso han tenido a nivel de ventas en la industria musical durante las últimas décadas, el rock y el pop (Jimi Hendrix, John Frusciante, Charly García, L. A. Spinetta, G. Cerati y J. Bieber).

\section{COMENTARIOS FINALES}

Resulta interesante destacar, en primer lugar, el amplio abanico de aspectos abarcados por las respuestas de lxs participantes en lo que atañe tanto a sus concepciones sobre ser músicx como a aquellas sobre ser músicx profesional, e, incluso, las diferentes, y hasta contrastantes, valoraciones que de una misma dimensión se han presentado. Este hecho sustenta la idea de que la heterogeneidad de la población de aspirantes a las carreras de Música de la FDA-UNLP no solo radica en el tipo de prácticas musicales que realizan (o no), sino que va mucho más allá de eso: tienen diferentes concepciones de lo que es ser músicx, ser músicx profesional, diversas maneras de verse a sí mismxs como músicxs o no, diferentes expectativas y motivaciones en relación a la carrera: de aprendizaje, de salida laboral, de proyectos a futuro (Pissinis, Peluffo y Martinez, 2020).

Por otro lado, resulta más llamativa aún la profunda reflexión de Ix participante que habló del rol social del músicx y de su responsabilidad civil y moral en contraste con los muchos otros casos que dijeron o insinuaron nunca antes haberse planteado interrogantes sobre qué es ser músicx o músicx profesional. Si bien es cierto que se trata de una persona de más edad que el promedio de Ixs entrevistadxs, con 
muchos años de estudio musical y tránsito por diferentes instituciones de formación profesional, tanto musical como de otras disciplinas, pareciera haber una cierta tendencia en muchxs aspirantes a las carreras de música a no reflexionar sobre estos aspectos, los cuales, creemos, son muy relevantes al momento de la elección de la carrera que van a estudiar. 


\section{BIBLIOGRAFÍA}

Ausubel, D.P., Novak, J.D. y Hanesian, H. (1978). “Educational Psichology. A Cognitive View". New York: Holt, Rinehart and Winston.

Blanco Guijarro, R. (2008). "Marco conceptual sobre educación inclusiva". En: La educación inclusiva: el camino hacia el futuro. Conferencia Internacional de Educación, 48 Reunión (pp. 5-14). Ginebra: UNESCO

Holguín, P. y Shifres, F. (2015). “Escuchar música al sur del Río Bravo: Desarrollo y formación del oído musical desde una perspectiva latinoamericana". En Calle 14,10(15) (pp. 40-53). Recuperado de http://sedici.unlp.edu.ar/handle/10915/52597

Lamont A. (2002). "Musical identities and the school environment". En R.A. MacDonald, D.J. Hargreaves, D. Miell (Eds.), Musical Identities (pp. 41-59). Oxford: Oxford University Press.

Malloch, S. y Trevarthen, C. (Eds.) (2009). “Communicative musicality: exploring the basis of human companionship". Oxford: Oxford University.

Martínez, I.C. (2018). "Music attending to linear constituent structures in tonal music". En Music \& Science 1. (pp. 1-17). Recuperado de: https://journals. sagepub.com/doi/pdf/10.1177/2059204318787763

Pissinis, J.F. y Martínez, I.C. (2018). “La kinesis instrumental y la conducción de las voces: construcción de conceptos musicales corporeizados". En N. Alessandroni y M.I. Burcet (Eds.), La experiencia musical. Investigación, interpretación y prácticas educativas. Actas del 13․ Encuentro de Ciencias Cognitivas de la Música. (pp. 361-376). Buenos Aires: SACCoM. Recuperado de http://sedici. unlp.edu.ar/handle/10915/74358

Pissinis, J.F. y Martínez, I.C. (2019a). "La Enseñanza de la Conducción de las Voces sobre la Base Multimodal de la Kinesis Instrumental". En: C. Giordano y G. Morandi (Comp.), Memorias de las $2^{\circ}$ Jornadas sobre la Práctica Docente en la Universidad Pública. La enseñanza universitaria a 100 años de la Reforma: legados, transformaciones y compromisos. (pp. 1350-1365). La Plata: Universidad Nacional de La Plata. Recuperado de http://sedici.unlp.edu.ar/handle/10915/79940

Pissinis, J.F. y Martínez, I.C. (2019b). “Educación musical universitaria inclusiva. Principales problemas para su análisis". En: M. A. Ordás, M. Tanco e I. C. Martínez (Eds.), Investigando la experiencia, la producción y el pensamiento acerca de la música. Actas de las Jornadas de Investigación en Música. (pp. 101-108). La

Plata: Laboratorio para el Estudio de la Experiencia Musical (LEEM), Facultad 
de Bellas Artes, Universidad Nacional de La Plata. Recuperado de http://sedici. unlp.edu.ar/handle/10915/74188

Pissinis, J.F., Peluffo, S. y Martínez, I.C. (2020). “Expectativas de ingresantes a carreras de música acerca de aprendizajes a realizar y proyectos profesionales a desarrollar". En C. Giordano y G. Morandi (Comp.), Memorias de las 30 Jornadas sobre la Práctica Docente en la Universidad Pública. El proyecto político académico de la Educación Superior en el contexto nacional y regional. (pp s/n). La Plata: Universidad Nacional de La Plata. Recuperado de http://sedici. unlp.edu.ar/handle/10915/106221

Salvador, K. (2014) "Identity and Transformation. (Re)claiming an Inner Musician". En C. Randles (Ed.), Music Education (pp. 215-232). New York: Routledge. Recuperado de https://www.researchgate.net/profile/Clint_Randles/publication/274894564_Music_Education_Navigating_the_Future/ links/5617a66408ae5d75aff6e004.pdf

Small, C. (1999). "El Musicar: Un ritual en el Espacio Social". En TRANS-Revista Transcultural de Música, 4(1), s/p. Recuperado de https://www.sibetrans.com/ trans/articulo/252/el-musicar-un-ritual-en-el-espacio-social

Valles, M.L., Martínez, I.C., Ordas, M.A y Pissinis, J.F. (2018) “Correspondence between the body modality of music students during the listening to a melodic fragment and its subsequent sung interpretation". En Parncutt, R. and Sattmann, S. (Eds.) ICMPC15/ESCOM10: Abstract book. Graz: Centre for Systematic Musicology, University of Graz. Recuperado de http://sedici.unlp.edu.ar/ handle/10915/70462

Vigotsky, L. (1978). "Mind in society: The development of higher psychological processes Development of Higher Psychological Processes". Cambridge: Harvard University Press 